\title{
Review
}

\section{Treatment of rheumatoid arthritis with biological agents - as a typical and common immune-mediated inflammatory disease}

\author{
By Tsutomu TAKEUCHI ${ }^{* 1, \dagger}$ \\ (Communicated by Tadamitsu Kishimoto, м.J.A.)
}

\begin{abstract}
Molecules involved in the disease process facilitated our understanding of pathogenesis of the disease with unknown etiology such as immune-mediated and inflammatory diseases. Moreover, the targeted therapies against the proposed molecular targets by biological agents provide enormous benefits to the patients and societies. Here, I will review recent progress of the biological treatment in the immune-inflammatory diseases by focusing on the rheumatoid arthritis, the disease characterized by persistent polyarthritis leading to joint destruction and disability with autoimmune features, as a role model.
\end{abstract}

Keywords: immune-mediated inflammatory diseases, rheumatoid arthritis, biologics, cytokines, therapy

\section{Introduction}

Molecular and cellular understanding of human diseases is critical for dissecting the mechanisms of pathogenesis and may provide a clue for developing innovative treatment. Among human diseases such as cancers, infections, and metabolic disease, there is a group of inflammatory diseases mediated by immunological mechanisms without apparent etiologies, called immune-mediated inflammatory diseases (IMIDs) including rheumatoid arthritis, inflammatory bowel diseases, and psoriasis. ${ }^{1) 2}$ While the human immune system involves highly complex and coordinated processes, it has been demonstrated that cytokines are overexpressed and T- and B-cells are accumulated in affected tissues in IMIDs. ${ }^{1,2)}$ These key players have been postulated as the potential targets for intervention. Indeed, novel therapeutic antibodies targeted against single molecules that are intimately associated with disease pathogenesis, have led to significantly better outcomes of diseases in clinical practice. ${ }^{3)-5)}$ The therapeutic antibodies are classified into the category of drugs, designated as

*1 Division of Rheumatology, Department of Internal Medicine, Keio University School of Medicine, Tokyo, Japan.

$\dagger$ Correspondence should be addressed: T. Takeuchi, Division of Rheumatology, Department of Internal Medicine, Keio University School of Medicine, 35 Shinanomachi, Shinjuku, Tokyo 160-8582, Japan (e-mail: tsutake@25.keio.jp). "biological agents" or "biologics", those are bioengineered to target specific proteins or nucleic acids. For instance, a common target of the inflammatory process is tumor necrosis factor (TNF), which is an inflammatory cytokine implicated in the pathogenesis of rheumatoid arthritis. Monoclonal antibodies against single cytokine, $\mathrm{TNF}$ alpha $(\mathrm{TNF} \alpha)$, have made revolutionary changes in treatment for a range of conditions such as rheumatoid arthritis (RA), inflammatory bowel disease, ankylosing spondyritis, and psoriasis (Table 1 ). The miracle achieved in the treatment of the IMIDs is reviewed here with a focus on RA, which is a typical and common IMID. ${ }^{6,7)}$

\section{Pathogenesis of rheumatoid arthritis as a typical and common immune-mediated inflammatory disease}

The immune system is beneficial to humans by protecting them from infections caused by bacteria or viruses. There are two major pathways of immunity: innate immunity as the first line of defense and acquired immunity that protects against specific pathogens. ${ }^{8)}$ There are unique diseases where inflammation is induced by the immune system, as seen in autoimmunity, and not by infection or malignancy. ${ }^{9}$ ) The immune-mediated inflammatory diseases include gout, where urate crystals formed in the body elicit inflammation through the innate immune system, and RA, where autoimmunity plays a central role in 


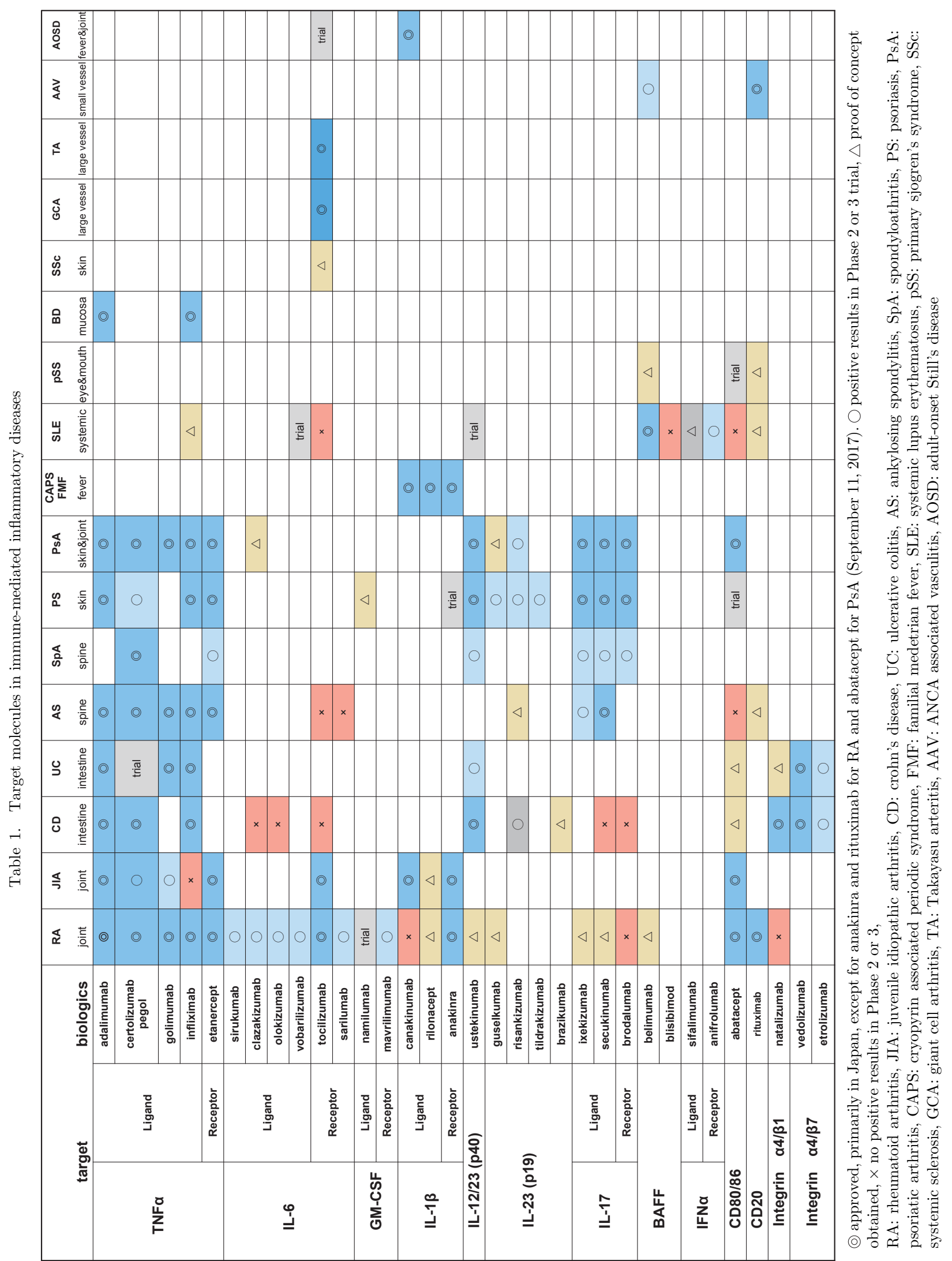




\section{a) Joint appearance (hands)}

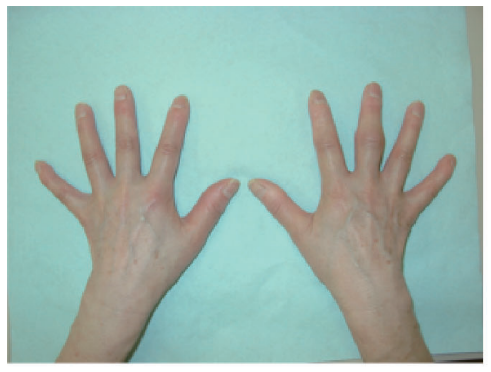

Early stage

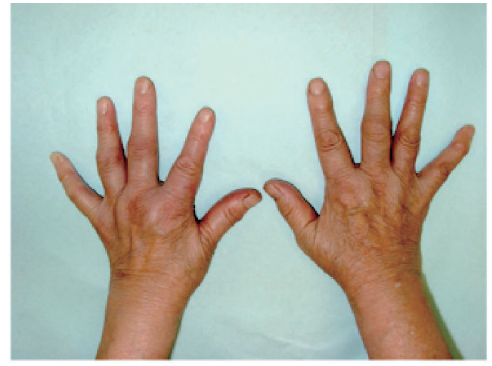

Established stage

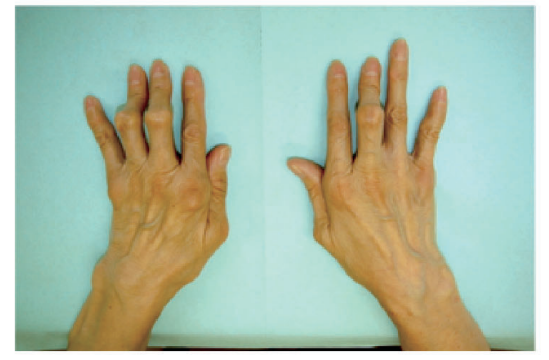

Late stage

\section{b) Pathological findings (synovial tissues)}

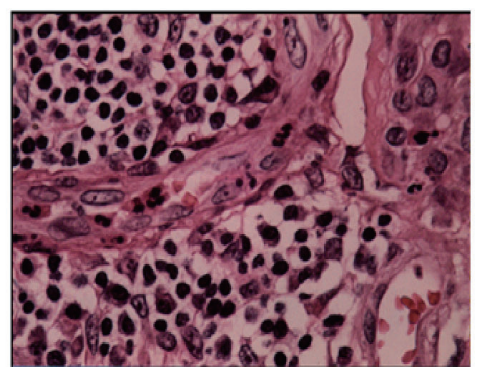

New vessel formation

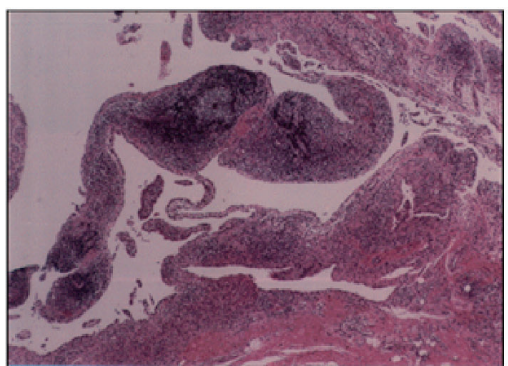

Lymphocyte infiltration

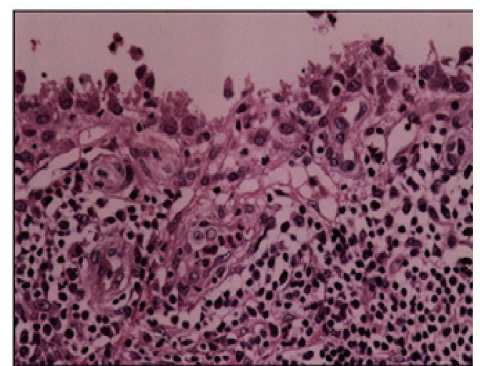

Synovial hypertrophy

Fig. 1. Clinical and pathological characteristics of rheumatoid arthritis.

perpetuating joint inflammation by breaking tolerance, which surveys and prevents the immune response against self (autoimmunity). RA affects $0.5 \%$ to $1.0 \%$ of the global population and is recognized as one of the common diseases in humans. RA is characterized by perpetual inflammation in the joint synovium leading to joint destruction and disability (Figure 1). ${ }^{4), 5)}$ With respect to the pathogenesis of RA, auto-reactive lymphocytes including $\mathrm{CD} 4+\mathrm{T}$ cells infiltrate into the joint through adhesion to the vessel wall by $\alpha 4 \beta 1$ integrinVCAM- 1 and $\alpha \mathrm{L} \beta 2$ integrin-ICAM- 1 as an initial step of inflammation of the joint. After infiltration into the joints, lymphocytes are further activated by antigen-presenting cells mounted with auto-antigens or by abundant extracellular matrix through receptors (Figure 2). An important requirement of $\mathrm{T}$ cell activation is co-stimulation through CD28, the absence of which leads to $\mathrm{T}$ cell anergy or death. Traditionally, $\mathrm{T}$ helper 1 cells are thought to be the $\mathrm{T}$ helper subset mediating the disease, but $\mathrm{T}$ helper 17 cells that produce IL-17A have been implicated in the pathogenesis. ${ }^{4), 5)}$ Activated $\mathrm{T}$ cells and B cells work together to interact with synovial cells to produce a large number of inflammatory mediators, including pro-inflammatory cytokines (TNF $\alpha$ and IL-6), induce matrix metallo-proteinases (MMP), promote synovial proliferation, and induce osteoclast differentiation (Figure 2). Three major cell types of endothelial cells, $\mathrm{T}$ and $\mathrm{B}$ cells, and synovial cells are present in the synovial tissues in patients with RA. ${ }^{4), 5)}$ Through interactions between these cells, pro-inflammatory cytokines such as TNF $\alpha$ and IL-6 are released, thereby facilitating the lowering of the threshold for pain and activating $\mathrm{T}$ cells, B cells, and macrophages (Figure 2). Activation of $\mathrm{T}$ cells is important in initiation and perpetuation of inflammation in RA. The hypothesis is corroborated by clinical efficacy of abatacept, an inhibitor against the co-stimulating molecule on $\mathrm{T}$ cells by substantial numbers of clinical trials. The inflammation is believed to result from or is perpetuated by autoantibodies such as anti-citrullinated peptide antibody and rheumatoid factors. ${ }^{5)} \mathrm{B}$ cells are thought to be responsible for the production of these autoantibodies. The pathogenic role of $\mathrm{CD} 20+\mathrm{B}$ cells has been validated by the efficacy of rituximab, a monoclonal antibody against CD20, in clinical trials. 


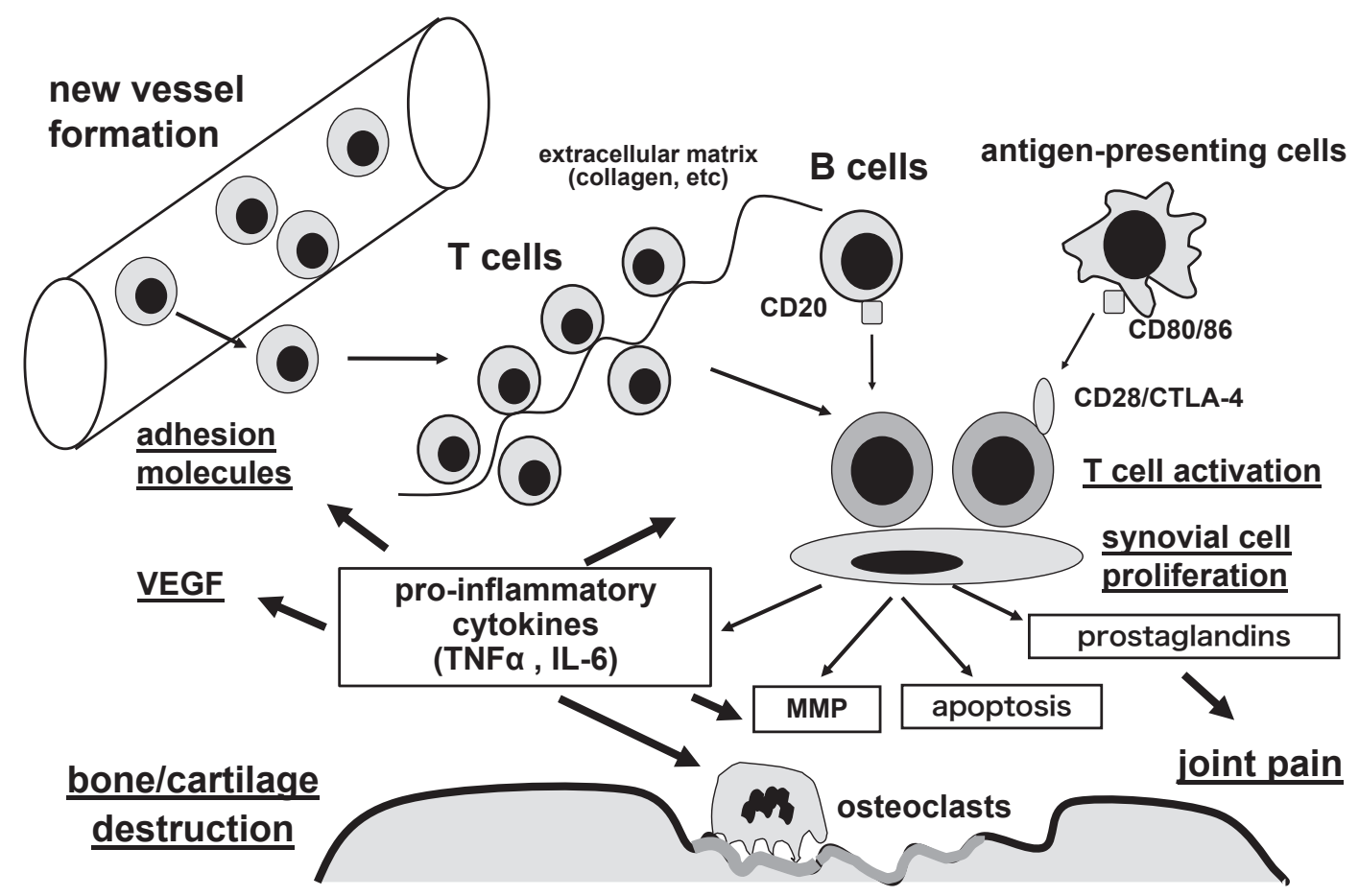

Fig. 2. Pathogenesis of rheumatoid arthritis.

\section{RA treatment in the past}

In the past, glucocorticoids and non-steroidal anti-inflammatory drugs were prescribed but they are largely symptomatic treatments and do not sufficiently inhibit joint destruction. Approximately three decades ago, half of RA patients with high disease activity were bed-ridden 10 years after such treatment and their life expectancy was shortened by 10 years compared with the general population. Thus, RA has been recognized as a typical example of an intractable immune-mediated inflammatory disease. Options had previously been limited for patients whose disease did not respond to traditional diseasemodifying anti-rheumatic drug (DMARDs) such as methotrexate.

In this regards, enormous efforts have been taken to develop new treatment options based on the molecular understanding for the key targets by biological agents (Figure 3).

Challenges toward miracle medicine: struggles with animal models and proof provided by clinical science

Several animal models of RA exist, including collagen-induced arthritis, collagen antibody-induced arthritis and TNF-alpha transgenic mice, which share characteristics with human RA. Although these models have limitations and do not encompass the complex mechanisms of RA, they are nevertheless invaluable in exploring disease pathogenesis and validating potential therapeutic strategies. The collagen-induced arthritis model, one of the induced arthritis models, suggested that IL-1 plays a central role in inducing arthritis, and antibodies against IL-1 and IL-1 knockout showed the dramatic prevention of the development of arthritis and inhibition of arthritis and joint destruction in the animal model. On the other hand, immunohistochemical staining of the synovial tissues from RA patients showed increased expression of $\mathrm{TNF} \alpha$ not only in synoviallining cells but also in subintimal tissues. ${ }^{5), 7)}$ In addition, it was demonstrated that the anti-TNF $\alpha$ monoclonal antibody could ameliorate collageninduced arthritis. ${ }^{10)}$ Given this evidence, there was much debate on whether IL- $1 \beta$ blockade is more potent than anti-TNF $\alpha$ in clinical efficacy and prevention of joint destruction in these animal models. Following these observations, IL-6 was reported to be an attractive target in arthritis. ${ }^{11)}$ Three agents - a chimeric monoclonal antibody against $\mathrm{TNF} \alpha$ infliximab; an IL-1 receptor antagonist, anakinra; and a humanized monoclonal antibody against the IL-6 receptor, tocilizumab - have been tested in clinical 


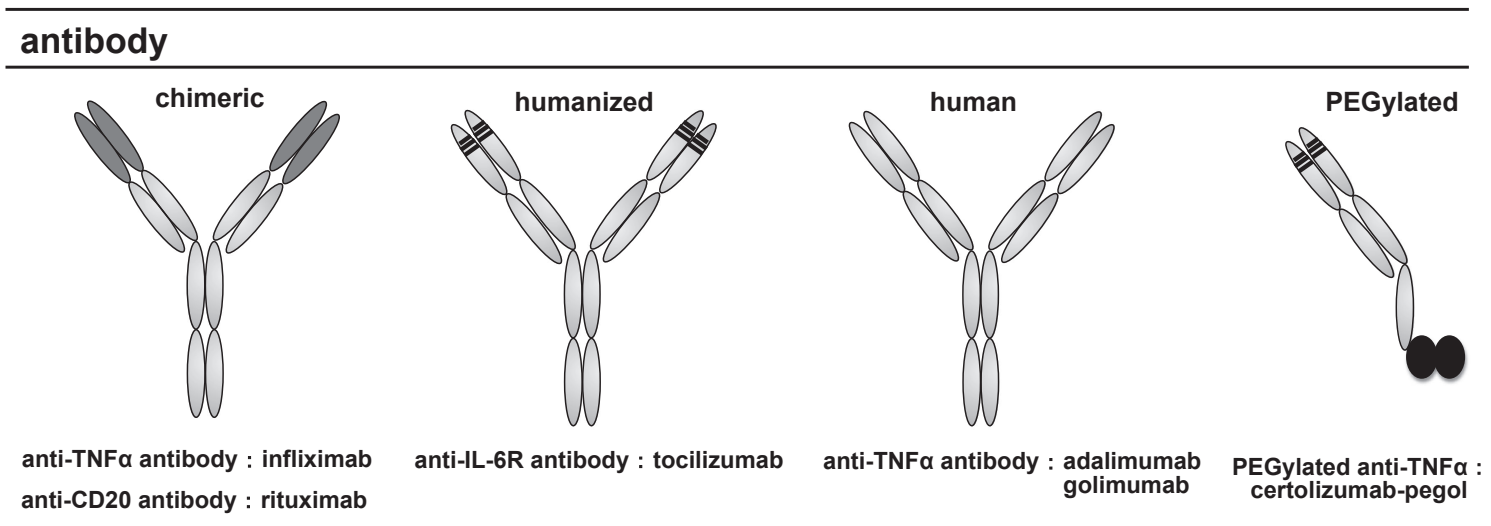

\section{receptor-IgGFc fusion proteins}

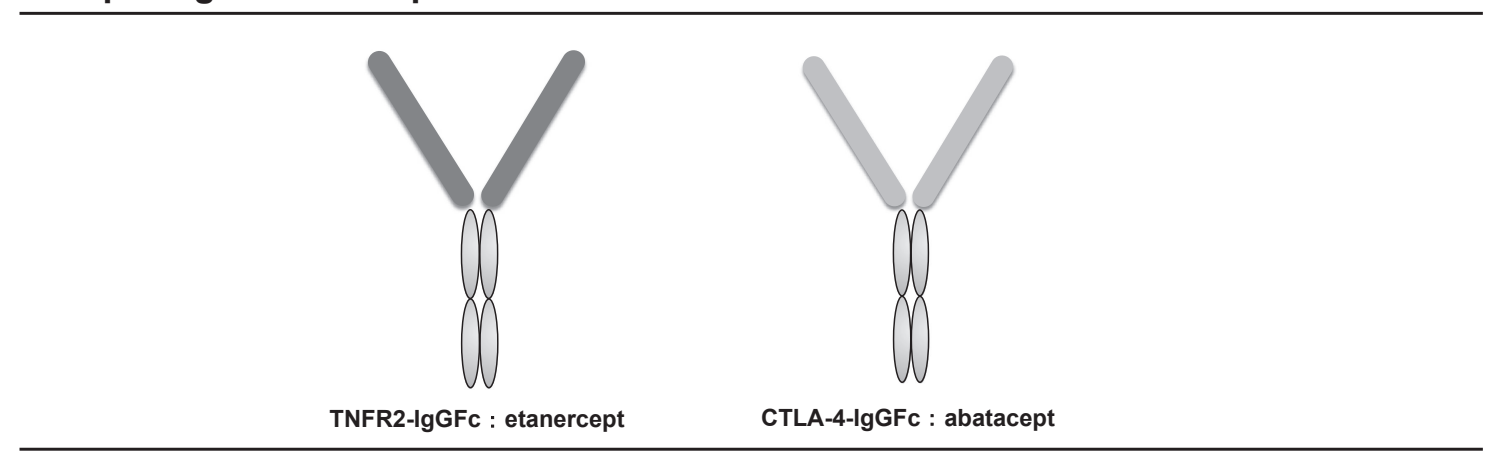

Fig. 3. Structure of biologics.

trials. ${ }^{11), 12)}$ The efficacy of infliximab ${ }^{13)}$ and tocilizu$\mathrm{mab}^{14)}$ was surprisingly excellent and they are widely used in clinical practice after completion of clinical trials and post-marketing clinical studies. ${ }^{6), 7)}$ Now, these agents against TNF $\alpha$ and the IL- 6 receptor are listed in the European League Against Rheumatism (EULAR) management guidelines and American College of Rheumatology (ACR) Guideline for the Treatment of RA, while IL-1 blockade is no longer positioned as a recommended biologic in RA treatment. ${ }^{15), 16)}$ This is because of its modest effect compared with other biologics, and the increased frequency of serious adverse events when used in combination with other biologics. Instead, IL-1 blockade is efficacious in auto-inflammatory diseases, including TNF receptor 1-associated periodic syndrome, as well as juvenile idiopathic arthritis, adultonset Still's disease, and gout (Table 1). These results again highlighted the importance of clinical science. Similar scenarios were observed with respect to IL-17 blockade, which was shown to ameliorate arthritis in mice models. However, the efficacy and safety of agents against IL-17 and IL-17 receptor failed to show results comparable with those of anti-
TNF and anti-IL-6 receptor. ${ }^{17)}$ It is interesting to note that the blockade of IL-17 exhibits significant efficacy in patients with spondyloarthritis, psoriasis, and psoriatic arthritis, indicating that IL-17 plays an indispensable role not RA, but in other major immune-mediate inflammatory diseases. ${ }^{17)}$ The difference between the results of basic research and the clinical trial can be derived from the difference in cell lines and mice strains used in basic research, and the study design with various genetic backgrounds for clinical studies. The comprehensive information from appropriately evaluated basic and early and exploratory clinical studies would lead to the efficient drug development.

\section{Cytokine cascade in RA}

Given the efficacy of biologics against different targets, one wonders whether patients who respond to one biologic targeting $\mathrm{X}$ will differentially respond to another targeting Y. Also, does group A of RA patients treated with anti-TNF overlap with group B of patients who respond well to anti-IL-6 receptor? One interesting hypothesis may answer part of these questions. Feldmann and Maini found that the level 


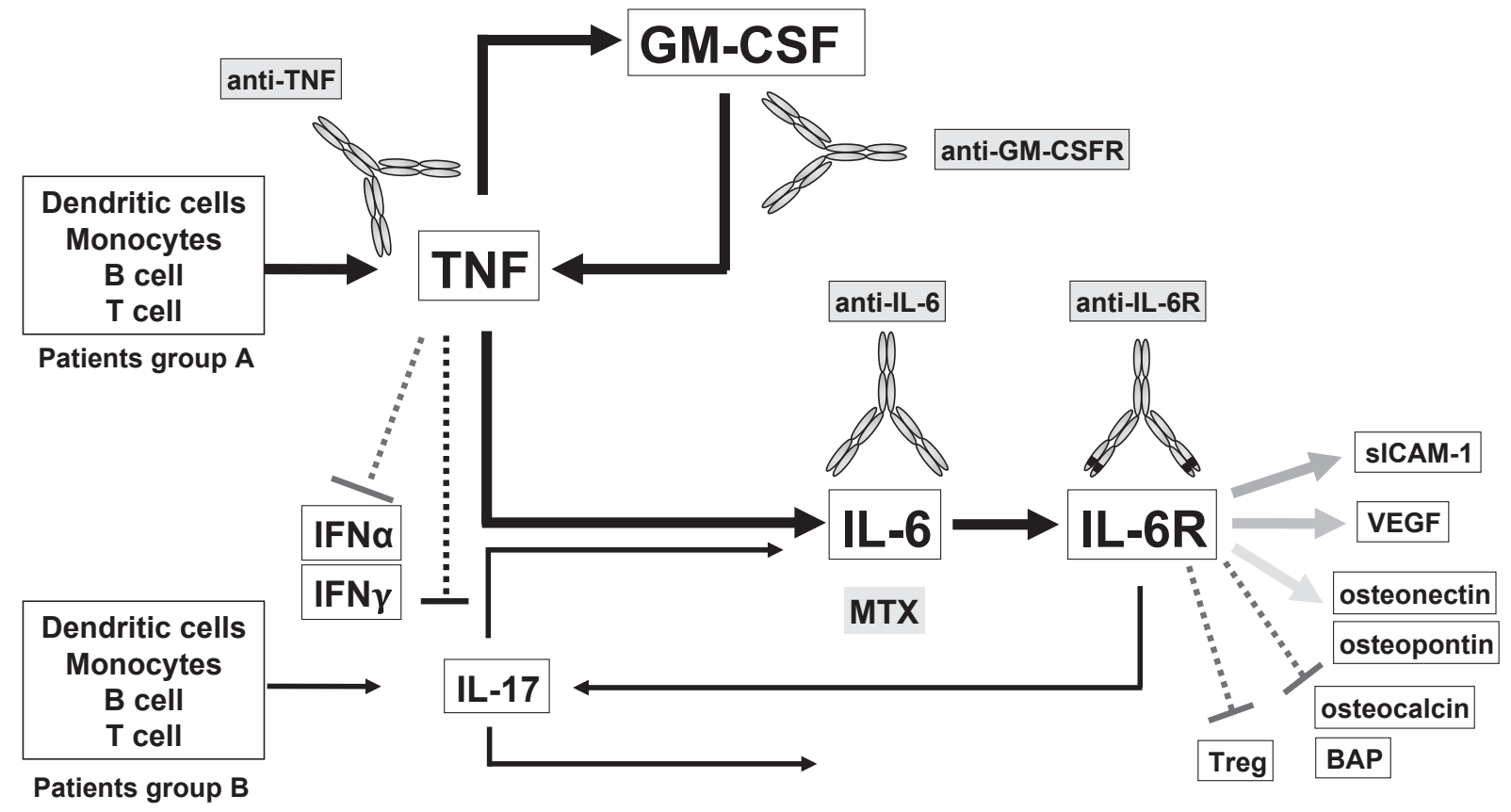

$\star$ TNF-IL-6 axis is playing a major role in RA

$\star$ TNF-independent IL-6 (IL-17-IL-6) or IL-6-independent axes may be contributing to a minor subset of RA patients

$\star$ The role and position of GM-CSF in the cytokine cascade should be determined

Fig. 4. Cytokine cascade hypothesis in rheumatoid arthritis.

of circulating IL-6 was significantly decreased several hours after infusing infliximab that target $\mathrm{TNF} \alpha$. This led to the introduction of a cytokine cascade hypothesis that $\mathrm{TNF} \alpha$ is located upstream of IL-6 and regulates the level of IL-6, so that blockade of TNF $\alpha$ leads to down-regulation of IL- 6 in patients with RA. ${ }^{10)}$ We confirmed this cytokine cascade hypothesis in the majority of RA patients and raised the possibility that a minor cascade from IL-17 to IL6 and a TNF and GM-CSF circuit exists on top of IL-6 (Figure 4). As shown in the figure, blockade of IL-6 signaling lead to a broad range of changes in biomarkers such as sICAM-1, VEGF, osteonectin, osteopontin, osteocalcin, and bone alkaline phosphatase. ${ }^{18)}$ Interestingly, regulatory $\mathrm{T}$ cells (Treg) are increased after treatment with tocilizumab, indicating that the inhibition of IL-6 signaling may cancel the transcriptional downregulation of Foxp3, a master transcriptional regulator of Treg. Alternatively, inhibition of IL-6 may affect the action of TGF- $\beta$ on inducing Treg. ${ }^{19)}$ Importantly, the increased proportion of Treg among CD4+ T cells is associated with the maintenance of clinical remission of RA in tocilizumab treatment. ${ }^{20)}$
In addition, our other study that analyzed data from the RISING study of infliximab suggested that inhibition of both TNF $\alpha$ and IL- 6 are essential for remission in RA patients. ${ }^{21)}$ Even if patients are prescribed infliximab, which blocks $\mathrm{TNF} \alpha$, the inhibition of IL- 6 is dispensable for clinical remission. ${ }^{22}$ Finally, blocking $\mathrm{T}$ cell co-stimulation by abatacept has shown to down-regulate circulating IL-6 levels in RA patients, ${ }^{23)}$ implying that $\mathrm{T}$ cells should play an important role in the cytokine cascade as an upstream position in RA (Figure 4).

\section{Timing of introduction of biologics: ideal positioning of biologics}

The timing of the introduction of biologics in RA treatment has been discussed extensively. In the 2015 ACR guideline, ${ }^{16)}$ initiation of a biologic or combination traditional DMARDs is recommended for patients with symptomatic early or established RA with moderate or high disease activity despite DMARD monotherapy. In the latest EULAR recommendations, ${ }^{15)}$ addition of a biologic to conventional DMARD is recommended if the treatment goal is not reached by 6 months. 


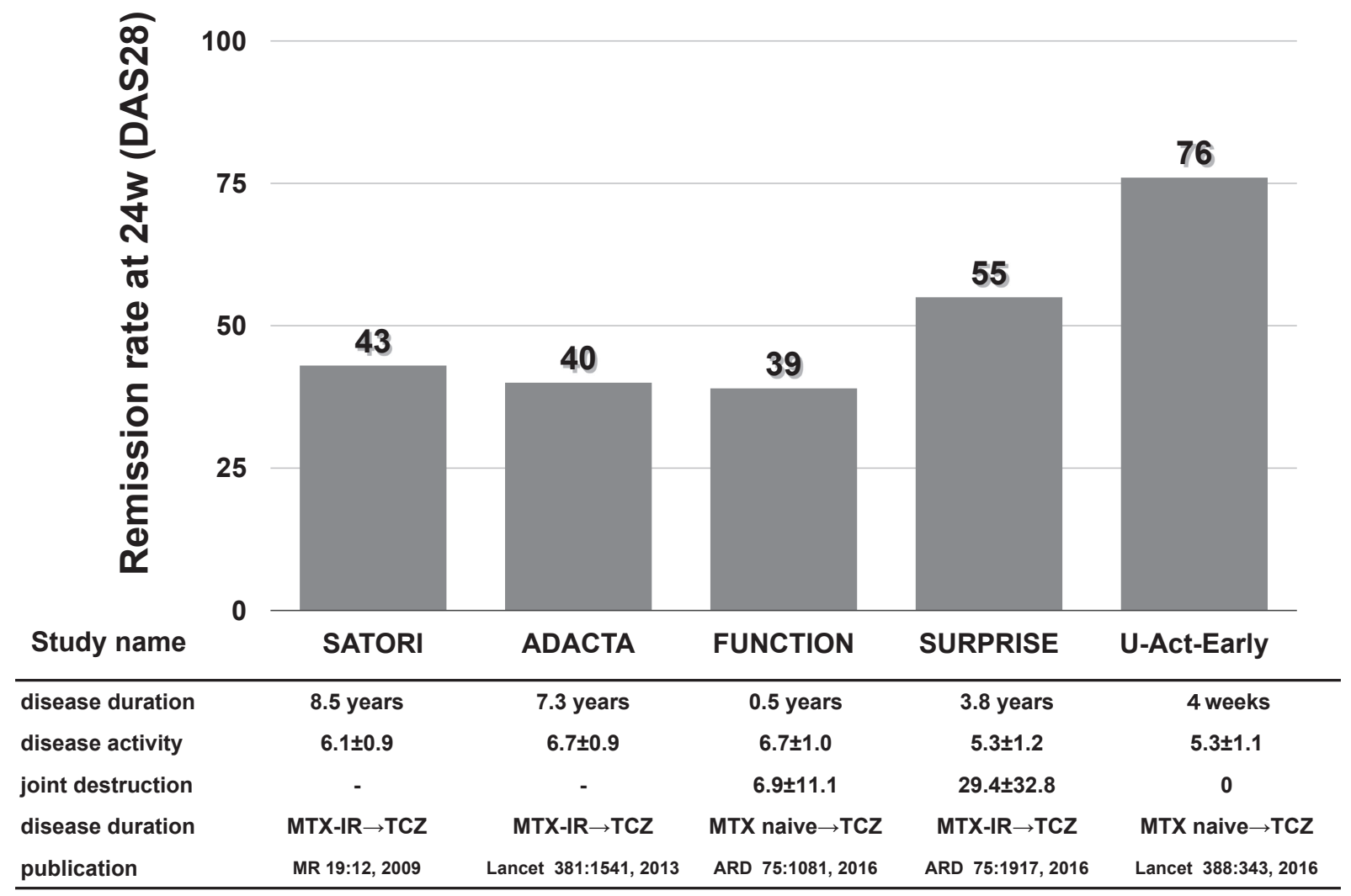

Fig. 5. Timing of introduction of biologics in rheumatoid arthritis. Clinical studies of tocilizumab monotherapy.

The newest clinical trials are currently testing which treatment strategy is the most useful to achieve remission in established RA in Japan (SURPRISE study) and very early RA in the Netherlands (U-ActEarly study). ${ }^{24), 25)}$ In the SURPRISE study, ${ }^{24)}$ adding tocilizumab to methotrexate (MTX) for those who respond inadequately to MTX is better than switching to tocilizumab as a significantly higher DAS28 remission rate was achieved at 6 months $(69.6 \%$ vs. $55.0 \%, \mathrm{p}<0.05)$. In the U-Act-Early study ${ }^{25)}$ disease duration of the patients was only 4 weeks after onset of initial symptoms and baseline joint destruction was zero, indicating that the recruited RA patients had very early stage disease. Sustained clinical remission was achieved in $80-90 \%$ of patients at 6 months in the tocilizumab monotherapy or tocilizumab + MTX combination groups. When we examined the clinical remission rate of these studies of tocilizumab monotherapy the collected data provided new information with regard to the timing of introduction of biologics in RA-initiation in those with short disease duration results in a greater likelihood of remission (Figure 5). One important clinical question here is whether we can stop biologics or not if we introduce biologics in earlier duration of the disease. There are several important clinical trials in early RA are reported to date, ${ }^{26)-29)}$ but it is too early to conclude that we can achieve drug free remission by introducing biologics earlier.

\section{Future perspectives}

There are several concerns regarding biologics in the treatment of immune-mediated inflammatory diseases. We need to establish useful biomarkers to predict or monitor efficacy and safety. ${ }^{6), 17), 30)}$ By analyzing the mechanism of action of the biologics, our knowledge is exponentially increased so that innovative tools to guide treatment such as cytokine levels at baseline, monitoring of the concentration of biologics, and DNA chip prediction will be developed that will contribute to personalized or precision medicine in the near future. On-target side effects such as infection should also be managed. The RA patients that are the most difficult to treat are those with co-morbidities such as bacterial or viral infection, interstitial pneumonitis, and other diseases of the major organs. Reactivation of latent infection in RA patients is also problematic. In addition, the 
socioeconomic burdens of biologics should be solved by facilitating the development of biosimilars, thereby further cost-cutting the original biologic by efficient bioengineering, and developing targeted synthetic DMARDs that can functionally replace biologics such as cytokine signaling inhibitors (JAK inhibitors, BTK inhibitors, and so on). There are still large unmet needs in RA treatment, and the search for new unique targets, targets located upstream in the cascade, and safer targets are continuing.

\section{Acknowledgements}

I would like to appreciate all staffs in Rheumatology division of Keio University School of Medicine for their hard work, all patients, as well as pharmaceutical companies to support clinical development in areas for the immune-mediated inflammatory diseases.

\section{References}

1) Kuek, A., Hazleman, B.L. and Ostor, E.L. (2007) Immune-mediated inflammatory diseases (IMIDs) and biologic therapy: a medical revolution. Postgrad. Med. J. 83, 251-260.

2) Davidson, A. and Diamond, B. (2001) Autoimmune diseases. N. Engl. J. Med. 345, 340-350.

3) Kopf, M., Bachmann, M.F. and Marsland, B.J. (2010) Averting inflammation by targeting the cytokine environment. Nat. Rev. Drug Discov. 9, 703-718.

4) McInnes, I.B. and Schett, G. (2011) The pathogensis of rheumatoid arthritis. N. Engl. J. Med. 365, 2205-2219.

5) Smolen, J.S., Aletaha, D. and McInnes, I.B. (2016) Rheumatoid arthritis. Lancet 388, 2023-2038.

6) Takeuchi, T. and Kameda, H. (2010) The Japanese experiences with biologic therapies for rheumatoid arthritis. Nat. Rev. Rheumatol. 6, 644-652.

7) Takeuchi, T. (2011) Revolutionary change in rheumatoid arthritis management with biological therapy. Keio J. Med. 60, 75-81.

8) Janeway, C.A., Travers, P., Walport, M. and Shlomchik, M.J. (2005) Immunobiology. (6th ed.). Garland Science.

9) Weinstein, J.S., Hernandez, S.G. and Craft, J. (2012) $\mathrm{T}$ cells that promote B-Cell maturation in systemic autoimmunity. Immunol. Rev. 247, 160-171.

10) Feldmann, M., Elliot, M.J., Woody, J.N. and Maini, R.N. (1997) Anti-tumor necrosis factor- $\alpha$ therapy of rheumatoid arthritis. Adv. Immunol. 64, 283350.

11) Kishimoto, T. (1997) Interleukin-6 from basic science to medicine: 40 years in immunology. Annu. Rev. Immunol. 23, 1-21.

12) Kishimoto, T. (2010) IL-6: from its discovery to clinical applications. Int. Immunol. 22, 347-352.

13) Elliott, M.J., Maini, R.N., Feldmann, M., Kalden,
J.R., Antoni, C., Smolen, J.S., Leeb, B., Breedveld, F.C., Macfarlane, J.D., Bijl, H. and Woody, J.N. (1994) Randomised double-blind comparison of chimeric monoclonal antibody to tumour necrosis factor alpha (cA2) versus placebo in rheumatoid arthritis. Lancet 344, 1105-1110.

14) Nishimoto, N., Yoshizaki, K., Miyasaka, N., Kazuhiko, Y., Kawai, S., Takeuchi, T., Hashimoto, J., Azuma, J. and Kishimoto, T. (2004) Treatment of rheumatoid arthritis with humanized anti-IL-6 receptor monoclonal antibody: a muticenter, double-blind, placebocontrolled trial. Arthritis Rheum. 50, 1761-1769.

15) Smolen, J.S. et al. (2017) EULAR recommendation for the management of rheumatoid arthritis with synthetic and biological disease-modifying antirheumatic drugs: 2016 update. Ann. Rheum. Dis. online March 6th.

16) Singh, J.A. et al. (2016) 2015 American college of rheumatology guideline for the treatment rheumatoid arthritis. Arthritis Rheum. 68, 1-25.

17) Semerano, L., Minichiello, E., Bessis, N. and Boissier, M.-C. (2016) Novel immuno-therapeutic avenues for rheumatoid arthritis. Trends Mol. Med. 22, 214-229.

18) Izumi, K., Kaneko, K., Hashizume, M., Yoshimoto, K. and Takeuchi, T. (2015) Baseline osteopontin levels predict the clinical effectiveness of tocilizumab, but not infliximab in biologic-naïve patients with rheumatoid arthritis: a single center prospective study at 1 year. PLoS One 10, e0145468.

19) Campbell, D.J. and Koch, M.A. (2011) Phenotypical and functional specialization of $\mathrm{FOXP}^{+}$regulatory T cells. Nat. Rev. Immunol. 11, 119-130.

20) Kikuchi, J., Hashizume, M., Kaneko, Y., Yoshimoto, K., Nishina, N. and Takeuchi, T. (2015) Peripheral blood $\mathrm{CD} 4^{+} \mathrm{CD} 25^{+} \mathrm{CD} 127^{\text {low }}$ regulatory $\mathrm{T}$ cells are significantly increased by tocilizumab treatment in patients with rheumatoid arthritis: increase in regulatory $\mathrm{T}$ cells correlates with clinical response. Arthritis Res. Ther. 17, R10.

21) Takeuchi, T., Miyasaka, N., Tatsuki, Y., Yano, T., Yoshinari, T., Abe, T. and Koike, T. (2011) Baseline tumor necrosis factor alpha levels predict the necessity for dose escalation of infliximab therapy in patients with rheumatoid arthritis. Ann. Rheum. Dis. 70, 1208-1215.

22) Takeuchi, T., Miyasaka, N., Tatsuki, Y., Yano, T., Yoshinari, T., Abe, T. and Koike, T. (2012) Inhibition of plasma IL-6 in addition to maintain efficacious trough level of infliximab was associated with clinical remission in patients with rheumatoid arthritis: analysis of the RISING Study. Ann. Rheum. Dis. 71, 1583-1585.

23) Weisman, M.H., Durez, P., Hallegua, D., Aranda, R., Becker, J.C., Nuamah, I., Vratsanos, G., Zhou, Y. and Moreland, L.W. (2006) Reduction of inflammatory biomarker response by abatacept in treatment of rheumatoid arthritis. J. Rheumatol. 33, 2162-2166.

24) Kaneko, Y. et al. (2016) Comparison of adding tocilizumab to methotrexate with switching to 
tocilizumab in patients with rheumatoid arthritis with inadequate response to methotrexate: 52 -week results from a prospective, randomised, controlled study (SURPRISE study). Ann. Rheum. Dis. 75, 1917-1923.

25) Bijlsma, J.W., Welsing, P.M.J., Woodworth, T.G. et al. (2016) Early rheumatoid arthritis treated with tocilizumab, methotrexate, and their combination (U-Act-Early): a multi-center, randomised, double-blind, double-dummy, strategy trial. Lancet 388, 343-355.

26) Emerly, P. et al. (2014) Sustained remission with eatenrcept tapering in early rheumatoid arthritis. N. Engl. J. Med. 371, 1781-1792.

27) Nishimoto, N. et al. (2014) Drug free REmission/low disease activity after cessation of tocilizumab (Actemra) Monotherapy (DREAM) study. Mod. Rheumatol. 24, 17-25.

28) Huizinga, T.W., Conaghan, P.G., Martin-Mola, E., Schett, G., Amital, H., Xavier, R.M., Troum, O., Aassi, M., Bernasconi, C. and Dougados, M. (2015)
Clinical and radiographic outcomes at 2 years and the effect of tocilizumab discontinuation following sustained remission in the second and third year of the ACT-RAY study. Ann. Rheum. Dis. 74, $35-43$.

29) Emery, P., Burmester, G.R., Bykerk, V.P., Combe, B.G., Furst, D.E., Barré, E., Karyekar, C.S., Wong, D.A. and Huizinga, T.W. (2015) Evaluating drug-free remission with abatacept in early rheumatoid arthritis: results from the phase $3 \mathrm{~b}$, multicentre, randomised, active-controlled AVERT study of 24 months, with a 12-month, double-blind treatment period. Ann. Rheum. Dis. 74, 19-26.

30) Takeuchi, T. (2017) Biomarkers as a treatment guide in rheumatoid arthritis. Clin. Immunol. 2017 Jul 20, pii: S1521-6616(17)30521-1. doi:10.1016/ j.clim.2017.07.019.

(Received Feb. 23, 2017; accepted June 7, 2017)

\section{Profile}

Tsutomu Takeuchi was born in 1955 in Nagano. He graduated from Keio University School of Medicine in 1980. After completion of post-graduate school of medicine at Keio University, he received Ph.D. degree in 1984. Subsequently, he was a research fellow at Dana-Farber Cancer Institute, Harvard Medical School in Boston from 1985 to 1986 for human immunology. He became Professor of Medicine at Saitama Medical University at 1998 and appointed as vice president of Saitama Medical University at 2004. He became Professor of Medicine at Keio University School of Medicine at 2009 and General Director of Keio University Hospital at 2013, served for 4 years. In August 2017, he is appointed as vice president of Keio University. He has done the pioneering work in the clinical development of biological agents in rheumatology and medicine. He received

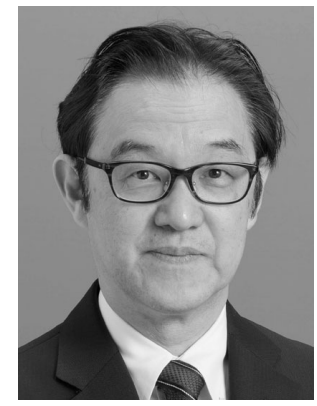
awards for his research, including the Japan College of Rheumatology, the Uehara memorial, the Naitoh Foundation, and the Waksman Foundation. He served as a chief investigator of the study group by Ministry of Health, Labor, and Welfare, Japan, and a principal investigator of Japanese and global clinical trials against Rheumatoid Arthritis and Systemic Lupus Erythematosus. He serves as a chairman of international committee of the Japanese College of Rheumatology and a member of scientific committee of European League Against Rheumatism. He is the President of Japanese Society for Inflammation and Regeneration and President of Japanese Society for Sjogren's syndrome. He was elected as the president of annual meeting of Asia-Pacific League against Rheumatology at 2020 and the president of 117th annual meeting of the Japan Society of Internal Medicine. 